\section{A systematic approach}

Sir, we would like to highlight the

4 importance of a methodical standardised approach to the assessment of the orthopantogram (OPG) radiograph.

A 40-year-old female patient was referred by her GDP to the department of oral and maxillofacial surgery with a presumed diagnosis of temporomandibular joint dysfunction. A routine investigation of an OPG examination was undertaken (Fig. 1). This revealed a radioopacity in the right posterior maxillary antrum (arrow) which prompted further investigations including a cone beam computerised tomograph and magnetic resonance imaging. The latter revealed an infiltrating mass of the right masticator space, infratemporal and pterygopalatine fossa and skull base. She has since undergone extensive surgical treatment for this.

It is important to reflect on the numerous pathologies that can present as trismus. These include trauma, non-odontogenic infections (including tetanus), odontogenic infections, temporomandibular disorders, medial pterygoid muscle injury, fibrosis of masticatory muscles and tumours. ${ }^{1}$ We would therefore reinforce the importance of a systematic and standardised approach in the assessment of radiographs - notably the OPG radiograph. Standardisation of procedures is an important approach to reducing medical error and is clearly documented in

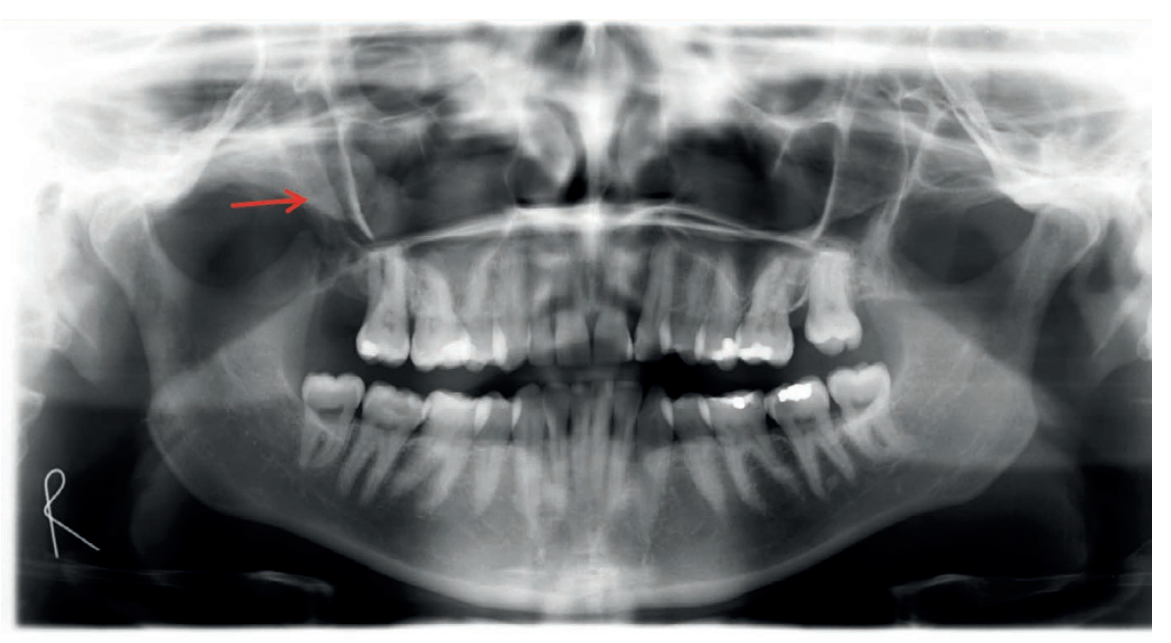

Fig. 1 OPG radiograph demonstrating a radio-opacity in the right posterior maxillary antrum (red arrow) 